\title{
36. COMMISSION DE LA THEORIE DES ATMOSPHERES STELLAIRES
}

\section{Report of Meeting, 26 August Ig64}

President: C. de Jager.

SECRETARY: A. B. Underhill.

The Draft Report was accepted in principle and all members of the Commission were urged to send corrections or minor additions to the President. The suggestions for President and Vice-President for the next three years made by the Executive Committee were adopted and the Organizing Committee was re-elected except for one change, Mustel being replaced by Sobolev. A revised list of members will be compiled.

The President reported briefly on the colloquia which had been organized by Commission 36 since the Eleventh General Assembly. A vote of thanks for organizing these stimulating meetings was moved by R. N. Thomas and passed unanimously.

Wrubel reported on his experience with the Astronomical Computation News Letter. There is a mailing list of about 100 and material for inclusion in the News Letter has been received from about 20 persons. Wrubel noted that the appearance of an issue usually generated a flurry of contributions and he wondered if it would be better to publish at irregular intervals whenever a few items had come in. He noted that it might be possible to enlarge the reports to contain descriptions of techniques. Persons desiring to be on the mailing list were asked to get in touch with Wrubel at the Department of Astronomy, University of Indiana. A vote of thanks was passed for Wrubel's work and he was asked to continue this service.

The following recommendations were adopted as a result of the review by the Working Group on Notation (Rudkjøbing and Underhill). This working group was dissolved.

\section{Recommendations concerning notations to be used in transfer theory}

(I) To denote a quantity that is a function of the wavelength or of the frequency, use a subscript $\nu$ if the spectral element is $d \nu$; use a subscript $\lambda$ if the spectral element is $d \lambda$.

(2) To denote a quantity that is independent of wavelength or of frequency or one whose wavelength dependence is unimportant in the context, use no subscript.

(3) To designate the monochromatic absorption coefficient use $\kappa$ with the appropriate subscript $\nu$ or $\lambda$ depending upon the element of spectral range being considered, $d \nu$ or $d \lambda$. Thus $\kappa_{\nu}$ would indicate the total absorption coefficient at $\nu$ due to all sources, both line and continuous spectrum. If it is desirable to designate these contributions separately, then in situ state that $\kappa_{v}$ represents the continuous absorption coefficient and that $l_{v}$ represents the line absorption coefficient. In this case one will not have a symbol for the combined value, but should carry both symbols, $\kappa_{\nu}$ and $l_{v}$, in the equations. If it is desired to indicate the source of the opacity use parenthesis, thus $\kappa_{\nu}\left(\mathrm{H}^{-}\right)$.

(4) To designate scattering coefficients use $\sigma$ with subscript $\nu$ or $\lambda$. We will reserve $\sigma$ with no subscripts for the radiation constant. To indicate the source of the scattering use parenthesis, thus $\sigma_{v}$ (e) for the electron scattering coefficient.

(5) The source-function should be designated by $S$, with or without subscript as required.

(6) The mean intensity (mean being taken over angle) should be designated by $J$. The notation $I$ is awkward and misleading in view of the common use of a bar over a quantity to designate an average value over frequency or wavelength. 
(7) The Planck function is designated by $B_{y}$; in astrophysical literature $B_{\nu}$ is per ster radian. The monochromatic radiation flux from a star is designated by $\pi F_{\nu}$. This flux may also be designated by $4 \pi H_{\nu}$.

(8) Various ways exist of defining temperature. It seems best to denote the effective temperature by $T_{\text {eff }}$ and the electron temperature by $T_{\mathrm{e}}$.

(9) The letter $\chi$ with suitable subscripts is well-established for excitation and for ionization potential. This practice should be continued.

(10) The order of the subscripts on a transition probability or like quantity are used to indicate the direction in which the transition occurs. The standard notation is to write the symbol for the initial state first and that for the final state last. There is no question of distinguishing between upper and lower levels, only between initial and final states. The product $g f$ is unchanged whether the transition is up or down. Here $g$ is the statistical weight of the initial state.

(I $\mathrm{r}$ ) The commission voted in favour of representing the depth in a spectral line by

$$
r_{\nu}(\mu)=\mathbf{I}-\frac{I_{\nu}(0, \mu)}{I_{\mathrm{c}}(0, \mu)}
$$

in the case of a pencil of radiation inclined to the normal by an angle $\theta=\cos ^{-1} \mu$ and in the case of fluxes by

$$
R_{\nu}=\mathrm{I}-\frac{F_{v}(0)}{F_{\mathrm{c}}(0)}
$$

